\title{
On ideal transforms of noetherian rings, II*
}

\author{
By \\ Jun-ichi NisHIMURA \\ (Received Nov. 12, 1978)
}

\section{Finiteness or integralness of global transforms of local rings and its applications}

Proposition (2.1) Let $\left(A, \mathfrak{m}_{1}, \cdots, \mathfrak{m}_{r}\right)$ be a semi-local ring with radical $\mathfrak{m}=$ $\mathfrak{m}_{1} \cdots \mathfrak{m}_{r}$ (containing a non-zero-divisor). Then

(2.1.1) $A_{\text {red }}(\mathfrak{n})$ is integral over $A(\mathfrak{n t})_{\text {red. }}$.

(2.1.2) $A_{\text {red }}(\mathfrak{m})$ is integral over $A_{\text {red }}$ if and only if $A(\mathfrak{m})$ is integral over $A$.

Let $\left(B, \mathfrak{n}_{1}, \cdots, \mathfrak{n}_{s}\right)$ be a semi-local A-algebra with radical $\mathfrak{n}=\mathfrak{n}_{1} \cdots \mathfrak{n}_{s}$, contained in $Q(A)$. Suppose $\operatorname{rad}(\mathfrak{m} B)=\mathfrak{n}$. Then

(2.1.3) $\quad B_{\text {red }}(\mathfrak{n})$ is integral over $A_{\text {red }}(\mathfrak{m})$ if and only if $B(\mathfrak{n})$ is integral over $A(\mathfrak{n})$.

Proof. Take any $\bar{x}$ in $A_{\text {red }}(\mathfrak{m})$. Since $\mathfrak{n}$ contains a non-zero-divisor in $A$, there exists $y$ in $Q(A)$ such that $\bar{y}\left(=\right.$ the image of $y$ in $\left.Q\left(A_{\text {red }}\right)\right)=\bar{x}$. As $\mathfrak{m}^{2} \bar{x}$ is contained in $A_{\text {red }}$ for some $\nu, \mathfrak{m}^{\nu} y$ is contained in $A+\operatorname{nil}-\operatorname{rad}(Q(A))$. Hence, if $\mathfrak{m}^{\nu}=a_{1} A+\cdots+a_{k} A, a_{j} y=y_{j}$ is integral over $A$. Let $A_{1}=A\left[y_{1}, \cdots, y_{k}\right]$, then there exists a non-zero-divisor $d$ in $A$ such that $\frac{1}{d} A$ contains $A_{1}$. Hence $y^{\mu}$ is in $A_{1}\left(\mathfrak{m} A_{1}\right) \subset \frac{1}{d} A(\mathfrak{m})$ for any $\mu$. Therefore $\bar{y}^{\mu}=\bar{x}^{\mu}$ is in $\frac{1}{d} A_{\text {red }}(\mathfrak{m})$ for any $\mu$. Since $A(\mathfrak{m})_{\text {red }}$ is noetherian [3], $\bar{x}$ is integral over $A(\mathfrak{m})_{\text {red. }}$. This proves (2.1.1).

(2.1.2) and (2.1.3) can be proved easily by (2.1.1).

Proposition (2.2) Let $\left(A, \mathfrak{n}_{1}, \cdots, \mathfrak{m}_{r}\right),\left(B, \mathfrak{n}_{1}, \cdots, \mathfrak{n}_{s}\right)$ be semi-local rings with common total quotient ring. If $B$ is integral over $A$, then $B(\mathfrak{n})$ is integral over $A(\mathfrak{m})$.

By (2.1.3), to prove Proposition (2.2), it is sufficient to prove the following:

Proposition $\left(2.2^{\prime}\right)$ Let $\left(A, \mathfrak{m}_{1}, \cdots, \mathfrak{m}_{r}\right),\left(B, \mathfrak{n}_{1}, \cdots, \mathfrak{n}_{s}\right)$ be semi-local domains with common field of quotients. If $B$ is integral over $A$, then $B(\mathfrak{n})$ is integral over $A(\mathfrak{m})$.

Proof. Let $B=\underset{\lambda}{\lim _{\lambda}} B_{\lambda}$, where $B_{\lambda}$ is a finite $A$-algebra (with radical $\mathfrak{n}_{\lambda}$ ).

* This article is the sequel of our paper: On ideal transforms of noetherian rings, I, Jour. Math. Kyoto Univ., 19 (1979), 41-46. 
Then, since $\mathfrak{n}$ is finitely generated, $B(\mathfrak{n})=\lim _{\vec{\lambda}} B_{\lambda}\left(\mathfrak{n}_{\lambda}\right)$. Therefore, as $B_{\lambda}\left(\mathfrak{n}_{\lambda}\right)$ is a finite $A(\mathfrak{m})$-module (cf. Lemma $(1.4)), B(\mathfrak{n})$ is integral over $A(\mathfrak{n} \mathfrak{t})$.

Proposition (2.3) Let $(A, \mathfrak{m})$ be a semi-local ring. Then

(2.3.1) the following are equivalent:

a) $A(\mathfrak{m})$ is integral over $A$.

b) $\operatorname{dim} \hat{A} / \hat{\mathfrak{p}}>1$ for any minimal prime ideal $\hat{\mathfrak{p}}$ in $\hat{A}$.

(2.3.2) the following are equivalent:

c) $A(\mathfrak{m})$ is a finite A-module.

d) $\operatorname{dim} \hat{A} / \hat{\mathfrak{p}}>1$ for any associated prime $\hat{\mathfrak{p}}$ of $\hat{A}$.

Proof. By Lemma (1.1), we may assume $A$ is complete.

1) a) $\Leftrightarrow \mathrm{b})$ : By (2.1.2), we may assume $A$ is reduced. The equivalence follows from Corollary (1.7) (cf. [6, Proposition 4]).

2) c) $\Rightarrow \mathrm{d}):$ i) Since $A$ and $A(\mathfrak{m})$ have common total quotient ring, there exists a canonical one-to-one correspondence between $\operatorname{Ass}(A)$ and $\operatorname{Ass}(A(\mathfrak{m}))$. Hence, to prove c) $\Rightarrow \mathrm{d})$, it is sufficient to show that $\operatorname{dim} A(\mathfrak{m}) / \mathfrak{p}^{\prime}>1$ for any $\mathfrak{p}^{\prime}$ in $\operatorname{Ass}(A(\mathfrak{m}))$.

ii) Since $A(\mathfrak{n})$ is a finite $A$-module, $A(\mathfrak{m})(\mathfrak{m})=A(\mathfrak{m})$. Hence $\operatorname{prof}\left(A(\mathfrak{m})_{\mathfrak{m}}\right)>1$ for any maximal ideal $\mathfrak{m}^{\prime}$ in $A(\mathfrak{m})$. Therefore $\operatorname{dim} A(\mathfrak{m} \mathfrak{r}) / \mathfrak{p}^{\prime}>1$ for any $\mathfrak{p}^{\prime}$ in $\operatorname{Ass}(A(\mathfrak{m}))$ (cf. [4, (15. F)]).

3) d) $\Rightarrow \mathrm{c})$ : i) Let $x$ be a non-zero-divisor of $A$ contained in $\mathfrak{m}$. Since $A$ is $(x$-adic) complete, to prove $\mathrm{d}) \Rightarrow \mathrm{c})$, it is sufficient to show that $A(\mathfrak{m}) / x A(\mathfrak{m})$ is a finite $A$-module and that $\bigcap^{\infty} x^{n} A(\mathfrak{m})=(0)$ (cf. [4, Lemma, p. 212]).

ii) $A(\mathfrak{m}) / x A(\mathfrak{n})$ is a finite $A$-module by THEOREM.

iii) Since $\operatorname{dim} A / \mathfrak{p}_{i}>1$ for any $\mathfrak{p}_{i}$ in $\operatorname{Ass}(A)(i=1, \cdots, s)$, there exists a non-maximal prime ideal $\mathfrak{q}_{i}$ such that $\mathfrak{q}_{i} \supset\left(\mathfrak{p}_{i}, x A\right)$ for any $i$. Hence the canonical homomorphism $A(\mathfrak{n} \mathfrak{t}) \rightarrow \prod_{i=1}^{s} A(\mathfrak{m})_{\mathfrak{q}_{i}}$ is injective. So, to prove $\bigcap^{\infty} x^{n} A(\mathfrak{m})=(0)$, it is sufficient to show that $\bigcap^{\infty} x^{n} A(\mathfrak{m})_{q_{i}}=(0)$ for any $i$.

iv) Since $\mathfrak{q}_{i}$ is non-maximal, $A(\mathfrak{m})_{q_{i}}=A_{q_{i}}$ by Lemma (1.1). Therefore $\bigcap^{\infty} x^{n} A(\mathfrak{m})_{\mathfrak{q}_{i}}=\bigcap^{\infty} x^{n} A_{\mathfrak{q}_{i}}=(0)$.

Corollary (2.4) Let $\left(A, \mathfrak{m}_{1}, \cdots, \mathfrak{m}_{r}\right)$ be a semi-local ring with radical $\mathfrak{m}=$ $\mathfrak{m}_{1} \cdots \mathfrak{m}_{r}$ (containing a non-zero-divisor). Then

(2.4.1) the following are equivalent:

a) $A(\mathfrak{m})$ is integral over $A$.

b) $\operatorname{dim} \hat{A} / \hat{\mathfrak{p}}>1$ for any minimal prime ideal $\hat{\mathfrak{p}}$ in $\hat{A}$.

(2.4.2) the following are equivalent:

c) $A(\mathfrak{m})$ is a finite A-module.

d) $\operatorname{dim} \hat{A} / \hat{\mathfrak{p}}>1$ for any associated prime $\hat{\mathfrak{p}}$ of $\hat{A}$.

Proposition (2.5) ([5, (33.11)], cf. [6, Proposition 7]) Let $\overline{\mathfrak{p}}$ be a height one prime ideal of the derived normal ring of a noetherian domain $A$. Then $\mathfrak{p}=\overline{\mathfrak{p}} \cap A$ 
is an associated prime ideal of a principal ideal of $A$ (i.e. prof $A_{p}=1$ ).

Proof. We may assume $A$ is local with maximal ideal $\mathfrak{p}$. Then $A(\mathfrak{p})$ is not integral over $A$ by Corollary (1.7). Hence $\hat{A}$ has a minimal prime ideal $\hat{\mathfrak{p}}$ such that $\operatorname{dim} \hat{A} / \hat{\mathfrak{p}}=1$ by (2.3.1). Therefore prof $A=1$ (cf. $[4,(15 . \mathrm{F})]$ ).

Theorem (2.6) Let $A$ be a noetherian ring and $I$ an ideal of $A$ (containing a non-zero-divisor $x$ ). Then

(2.6.1) the following are equivalent:

a) $A(I)$ is integral over $A$.

b) $A_{\mathfrak{p}}(\mathfrak{p})$ is integral over $A_{\mathfrak{p}}$ for any prime ideal $\mathfrak{p}$ of $A$ containing $I$.

(2.6.2) the following are equivalent;

c) $A(I)$ is a finite A-module.

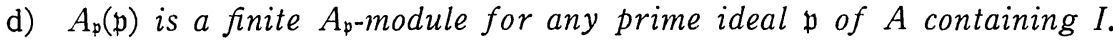

e) $A_{\mathfrak{p}}(\mathfrak{p})$ is a finite $A_{\mathfrak{p}}$-module for any prime $\mathfrak{p}$ in Ass $(A / x A)$ containing $I$.

Proof. 1) b) $\Rightarrow$ a): i) Take any $a$ in $A(I)$. We construct a sequence $\left(A_{i}, I_{i}\right)$ $(i=0,1,2, \cdots)$ of pairs of a finite $A$-algebra $A_{i}$ and a semi-prime ideal $I_{i}$ of $A_{i}$ such that $A_{0}=A, I_{0}=\operatorname{rad}(I), A_{i-1} \subset A_{i} \subset Q(A), a \in A_{i}\left(I_{i}\right), A_{i}$ satisfies b) for $I_{i}$ and that, if $I_{i-1} \cap A \subsetneq A, I_{i-1} \cap A \varsubsetneqq I_{i} \cap A(i=1,2, \cdots)$.

ii) Suppose $I_{i-1} \varsubsetneqq A_{i-1}$. Let $S=A_{i-1}-\bigcup_{j=1}^{s} \mathfrak{p}_{j}$, where $I_{i-1}=\mathfrak{p}_{1} \cap \cdots \cap \mathfrak{p}_{s}$. By inductive construction, $S^{-1} A_{i-1}\left(I_{i-1}\right)$ is integral over $S^{-1} A_{i-1}$ and $a \in A_{i-1}\left(I_{i-1}\right)$. Hence, there exists a finite $A_{i-1}$-algebra $A_{i}$ such that $a \in S^{-1} A_{i}$. Put $I_{i}=\operatorname{rad}\left(\left(A_{i}: a\right)_{A_{i}}\right)$. Then $a \in A_{i}\left(I_{i}\right), A_{i}$ satisfies b) for $I_{i}$ and $I_{i-1} \cap A \varsubsetneqq I_{i} \cap A$ by Proposition (2.2) and Lemma (1.1).

iii) As $A$ is noetherian, there exists a natural number $k>0$ such that $I_{k}=A_{k}$. Since $a \in A_{k}\left(I_{k}\right)=A_{k}, a$ is integral over $A$.

2) e) $\Rightarrow$ c): By Lemma (1.1), $A(I) \subset A_{x}$ and $A(I) \otimes_{A} A^{*}=A^{*}\left(I A^{*}\right)$, where $A^{*}$ is the $x$-adic completion of $A$. Hence, to prove e) $\Rightarrow \mathrm{c}$ ), we may assume $A$ is $x$-adic complete (cf. [4, Lemma 1, p. 7], [5, (8.9)]).

We claim: If $A$ satisfies e), $A(I) / x A(I)$ is a finite $A$-module and $\stackrel{\infty}{\cap}^{n} A(I)$ $=(0)$ (cf. [4, Lemma, p. 212]).

i) $\bigcap^{\infty} x^{n} A_{\mathfrak{p}}(I)=(0)$ for any prime ideal $\mathfrak{p}$ of $A$ containing $x$ (conseqently, as $A(I)$ is contained in $\left.Q(A), \bigcap^{\infty} x^{n} A(I)=(0)\right)$ : It is sufficient to show that, for any associated prime $\mathfrak{q}_{0}$ of $A$, there exists a prime $\mathfrak{q}$ of $A$, containing $\mathfrak{q}_{0}$ and $x$, such that $\bigcap^{\infty} x^{n} A_{q}(I)=(0)$.

Take a minimal prime $\mathfrak{q}$ of $\left(\mathfrak{q}_{0}, x A\right)$. Then $\mathfrak{q}$ is in Ass $(A / x A)$ by $[4$, Lemma 4, p. 99]. Suppose $q \supset I$, then $\operatorname{dim} A_{q} / q_{0}>1$ by (2.3.2). This contradicts the choice of $\mathfrak{q}$ (Hauptidealsatz), so $\mathfrak{q} \triangleright I$, then $A_{\mathfrak{q}}(I)=A_{\mathfrak{q}}$, and we get the assertion.

ii) $A(I) / x A(I)$ is a finite $A$-module: (We imitate Proof)

$\left.1^{\circ}\right)$ For any $a$ in $A(I)$, there exists a $k>0$ such that $a \in A x^{-k}+x A(I)$ : Because $A(I)$ is contained in $A_{x}$.

$\left.2^{\circ}\right)$ Let $J_{h}=\left(x^{h} A(I) \cap A, x A\right)$ be a descending chain of ideals of $A$. We 
claim :

$\left(^{*}\right)$ For $h \gg 0, J_{h}=x A$.

To prove $\left.{ }^{*}\right)$, it is sufficient to show :

$\left({ }^{*}\right)_{\mathfrak{p}}$ For $h \gg 0, x^{h} A(I)_{\mathfrak{p}} \cap A_{\mathfrak{p}} \subset x A_{\mathfrak{p}}$, whenever $\mathfrak{p}$ is in Ass $(A / x A)$.

If $\mathfrak{p} D I$, then $A(I)_{\mathfrak{p}}=A_{\mathfrak{p}}$. Hence $\left({ }^{*}\right)_{\mathfrak{p}}$ is clear. Suppose $\mathfrak{p} \supset I$. We may assume $\left({ }^{*}\right)_{\mathfrak{q}}$ is valid for any prime $\mathfrak{q}(\subseteq \mathfrak{p})$. Then $A_{q}(I) / x A_{q}(I)$ is a finite $A_{\mathfrak{q}}$-module (cf. Proof) and, if q contains $x, \bigcap^{\infty} x^{n} A_{q}(I)=(0)$. Hence we may assume, moreover, $A_{\mathfrak{q}}(I)$ is a finite $A_{\mathfrak{q}}$-module for any prime $\mathfrak{q}(\varsubsetneqq \mathfrak{p})$.

Now by assumption, there exists a finite $A$-algebra $B$ such that $B_{\mathfrak{p}}=A_{\mathfrak{p}}(\mathfrak{p})$. Then, since $A_{\mathfrak{p}}(\mathfrak{p})$ is a finite $A_{\mathfrak{p}}$-module, prof $\left(B_{\mathfrak{p}^{\prime}}\right)>1$ for any maximal ideal $\mathfrak{p}^{\prime}$ of $B_{\mathfrak{p}}$. Hence $\mathfrak{q}^{\prime} \cap A=\mathfrak{q} \varsubsetneqq \mathfrak{p}$ for any $\mathfrak{q}^{\prime}$ in Ass $\left(B_{\mathfrak{p}} / x B_{\mathfrak{p}}\right)$. Then, as $A_{\mathfrak{q}}(I)$ is a finite $A_{q}$-module, $B_{q}(I B)$ is a finite $B_{q^{\prime}}$-module. Therefore, for any given $i>0$, there exists a $h(i)$ such that $x^{h(i)} B_{\mathfrak{p}}(I) \cap B_{\mathfrak{p}} \subset x^{i} B_{\mathfrak{p}}$. Since $B_{\mathfrak{p}}$ is a finite $A_{\mathfrak{p}}$-module, there exists a $j>0$ such that $x^{j} B_{\mathfrak{p}} \cap A_{\mathfrak{p}} \subset x A_{\mathfrak{p}}$ (Artin-Rees). Therefore $x^{h(j)} A_{\mathfrak{p}}(I)$ $\cap A_{\mathfrak{p}} \subset x^{h(j)} B_{\mathfrak{p}}(I) \cap B_{\mathfrak{p}} \cap A_{\mathfrak{p}} \subset x A_{\mathfrak{p}}$.

$\left.3^{\circ}\right)$ By $1^{\circ}$ ) and $\left.2^{\circ}\right), A(I) / x A(I)$ is a finite $A$-module.

Proposition (2.7) (cf. [1, Proposition 1.1]) Let $(A, \mathfrak{m})$ be a local ring and $I$ an ideal of $A$ containing a non-zero-divisor $x$. Then

(2.7.1) the following are equivalent:

a) $A(I)$ is integral over $A$.

b) $\operatorname{dim} \hat{A} \hat{\mathfrak{q}} / \hat{\mathfrak{p}}>1$ for any prime ideal $\hat{\mathfrak{q}}$ of $\hat{A}$ containing $I \hat{A}$ and for any minimal prime $\hat{\mathfrak{p}}$ of $\hat{A}$ contained in $\hat{\mathfrak{q}}$.

(2.7.2) the following are equivalent:

c) $A(I)$ is a finite A-module.

d) $\operatorname{dim} \hat{A}_{\hat{\mathfrak{q}}} / \hat{\mathfrak{p}}>1$ for any prime ideal $\hat{\mathfrak{q}}$ of $\hat{A}$ containing I $\hat{A}$ and for any associated prime $\hat{\mathfrak{p}}$ of $\hat{A}$ contained in $\hat{\mathfrak{q}}$.

Proof. 0) To prove (2.7.1) and (2.7.2), we may assume $A$ is complete (cf. Lemma 1.1)).

1) a) $\Rightarrow$ b) follows from (2.6.1) and (2.3.1).

2) c) $\Rightarrow$ d) follows from (2.6.2) and (2.3.2).

3) Since a complete local domain satisfies the second chain condition (cf. $[5,(34.4)]), b) \Rightarrow$ a) follows from (2.6.1), (2.1.2) and Corollary (1.7) (cf. Lemma (1.1)).

4) Moreover, since any localization (with respect to a prime ideal) of a complete local domain is analytically unramified (cf. $[5,(36.3)]), d) \Rightarrow$ c) follows from (2.6.2) and 3) (cf. [4, (9.B)]).

\section{Derived normal rings of noetherian domains}

Proposition (3.1) (cf. [2, p. 74]) Let $A$ be a noetherian domain and $x$ a non-zero element of $A$. Suppose $A_{x}$ is normal and $\bar{A}_{\mathfrak{p}}$ is a finite $A_{\mathfrak{p}}$-module for any $\mathfrak{p}$ in Ass $(A / x A)$, then $\bar{A}$ is a finite A-module.

Proof. Let $M=\bar{A} \otimes_{A} A^{*}$, where $A^{*}$ is the $x$-adic completion of $A$. Since 
$A_{x}$ is normal, to prove the assertion, it is sufficient to show that $M$ is a finite $A^{*}$-module (cf. [4, Lemma 1, p. 7], [5, (8.9)]).

We claim: $M / x M(=\bar{A} / x \bar{A})$ is a finite $A^{*} / x A^{*}(=A / x A)$-module and $\bigcap^{\infty} x^{n} M$ $=(0)$ (cf. [4, Lemma, p. 212]).

1) $\bar{A} / x \bar{A}$ is a finite $(A / x A)$-module: (We imitate Proof)

$\left.1^{\circ}\right)$ For any a in $\bar{A}$, there exists a $k>0$ such that $a \in A x^{-k}+x \bar{A}$ : Because $A_{x}$ is normal.

$\left.2^{\circ}\right)$ Let $I_{h}=\left(x^{h} \bar{A} \cap A, x A\right)$. Then the descending chain of ideals $I_{h}$ stabilizes for some $h:$ By assumption, there exists a $m_{0}>0$ such that $x^{m} \bar{A}_{\mathfrak{p}} \cap A_{\mathfrak{p}}$ $\subset x A_{\mathfrak{p}}$ for any $m>m_{0}$ and for any associated prime $\mathfrak{p}$ of $A$ (Artin-Rees). Hence $I_{h}=x A$ for any $h>m_{0}$.

$3^{\circ}$ ) By Proof, $\left.1^{\circ}\right), 2^{\circ}$ ) give the assertion.

2) $\bigcap^{\infty} x^{n} M=(0)$ : Since $M$ is contained in the integral closure of $A^{*}$ in its total quotient ring, to get the claim, it is sufficient to show that $A^{*}$ is reduced (cf. [5, (33.10)]). This follows from the canonicol injection:

$$
A / x^{n} A \subset \underset{p \in A s s(A / x A)}{I} A_{p} / x^{n} A_{p} \quad \text { for any } n .
$$

Proposition (3.2) Let $A$ be a noetherian domain and $x$ a non-zero element of $A$. Suppose $A_{x}$ is normal and $A_{\mathfrak{p}}^{*}=\underset{n}{\lim } A_{\mathfrak{p}} / x^{n} A_{\mathfrak{p}}$ (=the $x$-adic completion of $A_{\mathfrak{p}}$ ) is reduced for any $\mathfrak{p}$ in $\operatorname{Ass}(A / x A)$. Then $\bar{A}$ is a finite A-module.

Proof. By Proposition (3.1), it is enough to show that $\bar{A}_{p}$ is a finite $A_{p}$ module for any $\mathfrak{p}$ in Ass $(A / x A)$.

If $h t \mathfrak{p}=1$, then $\bar{A}_{\mathrm{p}}$ is a finite $A_{\mathfrak{p}}$-module by [5, (32.2)]. Suppose ht $\mathfrak{p}>1$. We may assume that $A$ is local with maximal ideal $\mathfrak{p}$ and $\bar{A}_{\mathfrak{q}}$ is a finite $A_{\mathfrak{q}}$-module for any non-maximal prime $\mathfrak{q}$ of $A$. Let $\left(B, \mathfrak{n}_{1}, \cdots, \mathfrak{n}_{t}\right)$ be a well-branched overring of $A$. Suppose $\operatorname{dim} B_{n_{i}}>1$ iff $i \leqq s(\leqq t)$. Put $C=B_{S}$, where $S=B-\bigcup_{i \leq s} \mathfrak{n}_{i}$. Then $\left(C, \mathfrak{n}_{1}, \cdots, \mathfrak{n}_{s}\right)$ is a semi-local domain as in Proposition (1.2). We have

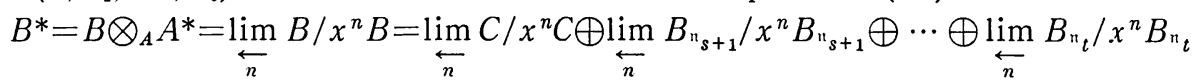
$=C^{*} \oplus B_{n_{s+1}}^{*} \oplus \cdots \oplus B_{n_{t}}^{*}$. Since $A^{*}$ is reduced, $B^{*}, C^{*}, B_{n_{j}}^{*}(j>s)$ are all reduced. Consequently, $\bar{B}_{n_{j}}$ is a finite $B_{n_{j}}$-module for any $j(>s$ ) (cf. [5, (32.2)]). Since $C^{*}$ is reduced and $C(\mathfrak{n}) / x C(\mathfrak{n})$ is a finite $C$-module (cf. Theorem), $C(\mathfrak{n})$ is a finite $C$-module (cf. Proof of Proposition (3.1)).

Hence, to prove $\bar{A}$ is a finite $A$-module, it is sufficient to show $\overline{C(\mathfrak{n})}$ is a finite $C(\mathfrak{n})$-module (cf. [4, Lemma 1, p. 7], [5, (8.9)]). Therefore, it is sufficient to prove $\overline{C(\mathfrak{n})_{\mathfrak{q}}}$, is a finite $C(\mathfrak{n})_{\mathfrak{q}^{\prime}}$-module for any $\mathfrak{q}^{\prime}$ in $\operatorname{Ass}(C(\mathfrak{n}) / x C(\mathfrak{n}))$. We have prof $\left(C(\mathfrak{n})_{\mathfrak{n}}\right)>1$ for any maximal ideal $\mathfrak{n}^{\prime}$ of $C(\mathfrak{n})$ by Proposition (1.2). Hence $\mathfrak{q}^{\prime} \cap A=\mathfrak{q}$ is non-maxmal in $A$. By assumption, $\bar{A}_{\mathfrak{q}}$ is a finite $A_{\mathfrak{q}}$-module. Therefore, $\overline{C(\mathfrak{n})_{\mathfrak{q}}}$, is a finite $C(\mathfrak{n})_{\mathfrak{q},}$-module.

Remark (3.3) (3.3.1) In Propositions (3.1) and (3.2), we cannot weaken the assumption "for any $\mathfrak{p}$ in $\operatorname{Ass}(A / x A)$ " by "for any $\mathfrak{p}$ in minimal Ass $(A / x A)$ " (cf. [1, Proposition 3.3]). 
(3.3.2) (Seydi) If $\left(A_{\mathfrak{m}}\right)^{(1)}=\underset{\substack{h t p=1 \\ p \subset \text { m }}}{A_{p}} A_{\text {is }}$ a finite $A_{\mathrm{r}}$-module for any maximal ideal $\mathfrak{m}$ of $A$, then we can weaken the assumption "for any $\mathfrak{p}$ in $\operatorname{Ass}(A / x A)$ " in Propositions (3.1) and (3.2) by "for any $\mathfrak{p}$ in minimal Ass $(A / x A)$ " (Proof is easy).

\section{Department of Mathematics KYOTO UNIVERSITY}

\section{References}

[1] D. Ferrand-M. Raynaud: Fibres formelles d'un anneau local noethérien, Ann. Sci. Ecole Norm. Sup. (4) 3 (1970), 295-311.

[2] H. Kurke, G. Pfister und M. Roczen: Henselsche Ringe und algebraische Geometrie, VEB Deutscher Verlag der Wissenschaften, 1975.

[3] J. Matijevic: Maximal ideal transforms of noetherian rings, Proc. Amer. Math. Soc. 54 (1976), 49-52.

[4] H. Matsumura : Commutative algebra, Benjamin, 1970.

[5] M. Nagata: Local rings, Interscience, 1962.

[6] J. Nishimura: Note on integral closures of a noetherian integral domain, Jour. Math. Kyoto Univ. 16 (1976), 117-122.

[7] J. Querré: Sur un théorème de Mori-Nagata, C. R. Acad. Sc. Paris, t. 285 (1977), 323-324.

[8] H. Seydi: Sur la transformation de Nagata (preprint). 